\title{
EVALUATION OF NANO-GRAPHENE BASED SANDWICH AND DOT-ELISA AS PROMISING TECHNIQUES FOR DIAGNOSIS OF HUMAN INTESTINAL GIARDIASIS
}

\author{
By \\ FATMA M. EL-LESSY ${ }^{1}$, KAREMAN M. ZEKRY1*, AISHA T. HASAN ${ }^{1}$ \\ AND IBRAHIM R. ALY2
}

Department of Medical Parasitology, Faculty of Medicine for Girls ${ }^{1}$, Al-Azhar

University, Cairo, and Department of Parasitology, Theodore Bilharz Research

Institute'2, Imbaba P.O. Box 30, Giza, Egypt

( ${ }^{\star}$ Correspondence:kimokono630@yahoo.com)

\section{Abstract}

Giardia intestinalis nowadays is recognized as the most common parasitological cause of diarrhea, with 280 million infections per year. Microscopic examination of faecal samples has the advantage of low cost and the ability to simultaneously identify other parasitic infections. However, analysis of single stool sample and the skill of the microscopist can affect the accuracy of detection.

As an attempt to improve the sensitivity of laboratory diagnosis of giardiasis, the present study aimed to evaluate the diagnostic performance of different parasitological techniques (Mini Parasep, MIFC and direct smear) and to evaluate a novel antigen capture immunoassays based on IgG polyclonal antibody conjugated with nanoparticles (Nano graphene based Sandwich ELISA and Dot-ELISA) for detection of Giardia antigen in stool samples. A total of 96 human stool samples were collected and classified into three groups according to stool examination results, (GI), 61 Giardia infected patients, (GII), 20 samples collected from patients infected with other parasites and (G III), 15 healthy individuals (negative control).

In the current study, Giardia antigen detection was carried out by several steps including preparation of Giardia antigen, production, purification and labeling of rabbit anti-Giardia IgG polyclonal antibodies.

The Mini Parasep was the best followed by MIFC and direct smear. The study demonstrated that Nano Sandwich ELISA was higher than Traditional Sandwich ELISA regarding sensitivity, PPV, NPV and diagnostic accuracy with statistically significant difference between them, while specificity in Traditional Sandwich ELISA was higher than Nano Sandwich ELISA. Dot ELISA and Nano Dot ELISA had the same sensitivity, while Dot ELISA was higher than Nano Dot ELISA regarding specificity, PPV, NPV and diagnostic accuracy without significant difference. This means that the use of graphene nanoparticles improved the diagnostic testing of human giardiasis.

Key words: Giardiasis, Giardia, Sandwich ELISA, Dot-ELISA, Graphene Nanoparticles, Diagnosis, Mini Parasep.

\section{Introduction}

Giardia intestinalis (G. intestinalis or G. duodenalis, G. lamblia) is a flagellate intestinal protozoan that infects man and many animal species (Adam, 2001; Bey-han and Cengiz, 2017). It is considered one of the major causes of enteritis in humans worldwide (Klotz and Aebischer, 2015). It is particularly common in developing countries, where poor sanitation and bad hygiene are major problems. The prevalence of infection was $2 \%-5 \%$ in developed countries and up to $30 \%$ in developing countries. Additional- ly, the highest percent of infection occurs in children younger than 10 years old (Caccio and Sprong, 2011; Diagbouga et al, 2017). Giardiasis is still a significant public health problem. It is included in the 'Neglected Diseases Initiative' of the World Health Organization (WHO) in 2004 (Diagbouga et al, 2017).

Giardiasis is endemic in Egypt (Eissa and Amer, 2010). It was considered one of the most common causes of chronic diarrhea in infants and children (13.6\%). It plays an important role in stunting and cognitive im- 
pairment in poorly nourished children (ElDeeb and Abdel-Hamid, 2012).

The clinical manifestations of giardiasis vary from asymptomatic infection to acute or chronic diarrhea with abdominal pain, flatulence, weight loss and malabsorption that can last for several months (Romero et al, 2015).

Laboratory diagnosis of giardiasis depends generally on microscopic detection of cysts and/or trophozoites of the parasite in stool samples (Hanson and Cartwright, 2001; Beyhan and Cengiz, 2017). It is effective, cheap and has the advantage of detecting large variety of parasitic infections (Mendonça et al, 2017).

However, sensitivity is poor when only a single sample is analyzed, particularly in low parasite density or intermittent excretion of cysts. Microscopic examination must be performed on three stool samples within 3-5 days to increase the sensitivity (El-Nahas et al, 2013).

The antigen detection immunoassays such as Enzyme Linked Immunsorbant Assay (ELISA) and immunochromatography (IC) were developed to detect Giardia antigen in feces (Arora and Arora, 2015). ELISA is a rapid, sensitive and economic method for detection of specific antigens in stool and confirmation of certain infection (Barazesh et al, 2010).

Nanotechnology is important for medical diagnosis. The development of nanotechnology architecture and materials could potentially extend sub-cellular and molecular detection beyond the limits of conventional diagnostic modalities (Hu et al, 2011). Nanotechnology may improve the sensitivity, specificity, speed, cost and convenience of diagnostic tests. Furthermore, nanotechnology has also opened up the possibility of other screening strategies (Hegazy et al, 2015).

In the past few years, graphene and its derivatives have attracted considerable attention because of their excellent thermal and electrical conductivity, biocompatibility, high specific surface area and various surface oxygen-containing groups (Wang et al, 2017). Mechanically, graphene also appears to be one of the strongest materials ever tested, with high elasticity, flexibility and adaptability to flat or irregular surfaces (Liu et al, 2012).

Scientists start to think about tuning the properties of graphene by controlling the size of graphene. Thus, nano-sized graphene (nanographene, NG) was developed and attracted attention (Dai et al, 2018). The sensitivity of laboratory diagnosis of $G$. intestinalis infection was improved by including alternative diagnostic procedures which are more rapid and reliable (El-Nahas et al, 2013).

The present study aimed to compare the diagnostic performance of different parasitological methods and to evaluate Nano graphene based Sandwich ELISA and DotELISA for detection of Giardia antigen in stool samples of infected patients as promising non-invasive techniques to diagnose giardiasis.

\section{Materials and Methods}

Study population: This study was performed from December 2016 to April 2018. It was carried out on patients complaining of gastrointestinal symptoms suggestive of intestinal giardiasis. Fresh fecal samples were collected from patients attending the outpatient clinics of Al-Zahraa University hospital, Abo El Reesh Pediatric hospital and Theodor Bilharz Research Institute (TBRI). Verbal consent was taken from each one.

This study was conducted on 96 stool samples. Grouping of the participants was done as follows based on stool examination (direct and concentration) results: GI: 61 patients positive to $G$. intestinalis. This group was subdivided into: GIa: 49 patients positive to Giardia cyst and GIb: 12 patients positive to Giardia trophozoite. GII: 20 patients harboring other parasites than $G$. intestinalis: 5 patients infected with Blastocystis hominis, 2 patients infected with Hymenolepis nana and 13 patients infected with Entamoeba histolytica/dispar. GIII: 15 appar- 
ently healthy individuals free from giardiasis and other parasitic infections served as negative control.

The fecal samples were collected in clean wide mouth containers. All samples were divided into 3 portions as soon as they were received: a- A small part of each specimen for direct smear examination, b- Another part preserved in 10\% formalin for merthiolate iodine formaldehyde concentration method (MIFC) and Mini Parasep method, and c- The majority of fecal samples were freezed at $-20^{\circ} \mathrm{C}$ for immunological techniques (Traditional and Nano based Sandwich ELISA \& Dot ELISA).

Parasitologic examinations: A- Direct smear method (Garcia, 2007): A sample of about $2 \mathrm{mg}$ of stool was taken and simply emulsified in a drop of normal saline, placed on a dry clean glass slide using wooden applicator. Cover slip was applied to make a thin film free of air bubbles, examined by 10X \& 40X by light microscope. Presence or absence of Giardia cysts/trophozoite was recorded. B- Merthiolate iodine formaldehyde concentration method (MIFC) (Blagg et al, 1955): Approximately $1 \mathrm{gm}$ of fecal specimen was emulsified in a tube containing $5 \mathrm{ml}$ of merthiolate iodine formaldehyde (MIF) mixed well and filtered in other cup, and followed by addition of $7 \mathrm{ml}$ ether. The prepared specimen was centrifuged for $5 \mathrm{~min}$ at $3500 x$. A drop of sediment was put on a slide, covered and examined under light microscope, $(\mathrm{MIF}=\mathrm{a}$ mixture of 2 solutions with ratio $4: 1$. A composed of $0.1 \%$ merthiolate, $36-40 \%$ formaldehyde, glycerin and distilled water; B composed of potassium iodide, iodine and distilled water). CMini Parasep concentration test (APACOR Ltd, England) after Sanprasert et al. (2016): The Mini Parasep ${ }^{\circledR}$ solvent free (Mini Parasep $^{\circledR} \mathrm{SF}$ ) tubes and the sedimentation cones (fig.1) were labelled with the specimen identification numbers. The procedure was used according to manufacturer's directions. The stool sample was introduced to the mixing tube using a spoon on the end of the filter. It was then mixed with $3.3 \mathrm{ml}$ of $10 \%$ formalin in mixing tube. Mini Parasep tube was sealed by screwing in filter/ sedimentation cone unit, vortexed to emulsify its content with sedimentation cone pointing upwards, and then inverted and centrifuged at $200 \mathrm{xg}$ for 2 min. The mixing chamber and filter were then unscrewed and the suspension in the sedimentation cone was discarded. The sediment was microscopically examined using physiological saline.

NB: Comparison of diagnostic performance of direct smear, Mini Parasep \& MIFC used for detecting Giardia cyst in stool samples of GIa was done for only 43 stool samples due to inadequate quantity of other samples to perform all techniques.

Preparation and purification of Giardia antigen by two-phase sucrose gradient technique (Moss et al, 1991): The samples chosen for cyst purification were heavily infected with high number of the cysts (more than 8 cysts in each microscopic field with $40 \mathrm{X}$ ), also free from any other gastrointestinal parasites or yeast contamination.

Samples were diluted with distilled $\mathrm{H}_{2} \mathrm{O}$ and filtered through four layers of gauze to remove the course materials. The filtrate was centrifuged at $800 \times g$ for $5 \mathrm{~min}$. The supernatant was eliminated and the deposit was washed again. In the first phase the pellet was re-suspended in $20 \mathrm{ml}$ of distilled $\mathrm{H} 2 \mathrm{O}$ and divided into several aliquots of $5 \mathrm{ml}$ Each of these aliquots was placed over $3 \mathrm{ml}$ of cold $1.5 \mathrm{M}$ sucrose and centrifuged at $600 \times g$ for $10 \mathrm{~min}$. The interfaces were removed carefully. In the second phase, the obtained portions were dissolved in distilled $\mathrm{H} 2 \mathrm{O}$ and the suspensions were added to 0.75 $\mathrm{M}$ sucrose solution followed by centrifugation at $1500 \times g$ for $10 \mathrm{~min}$. So, cysts settled at tubes' bottom. The entire procedure was conducted at room temperature. The purified cysts were then stored at $-40^{\circ} \mathrm{C}$.

Protein content determination: Protein content determination was based on Bradford dye-binding procedure which was dependent on the color change of Coomassie brilliant 
blue G-250 dye in response to various concentrations of proteins (Bradford, 1976).

Assessment of reactivity of Giardia antigen by triple rapid diagnostic test (Cer-Test Biotec S.L. Spain): CerTest Crypto+ Giard$i a+$ Entamoeba one step combo card test (Fig. 2), a colored chromatographic immunoassay for simultaneous qualitative detection of Cryptosporidium, Giardia, Entamoeba histolyticaldispar in stools.

Card test, test sample (Giardia antigen) $\&$ stool collection tubes with diluent were allowed to reach room temperature (15$30^{\circ} \mathrm{C}$ ) prior to testing. The rapid test was used according to manufacturer's directions. 1- Sufficient test sample quantity (approx. $125 \mathrm{mg}$ ) was picked up by stick and added to the stool collection tube with diluent. Tube was closed and shaked well in order to assure good sample dispersion. 2- Cer-Test Crypto+Giardia+Entamoeba combo card test was removed from sealed bag just before use. 3- Stool collection tube was opened to dispense 4 drops in each of circular windows marked with the letter A, B \& C. 4Results read within 10 min.

Production and purification of polyclonal antibodies (PAb): 1. Immunization of the rabbit for production of polyclonal antibodies: Production of $\mathrm{pAb}$ was obtained by immunizing New Zealand white rabbit with purified Giardia antigen through primary dose in the form of intramuscular injection at two sites 1ml Giardia antigen mixed 1:1 with complete Freund's adjuvant (CFA), (Sigma) this was followed by three booster doses, each was $0.5 \mathrm{ml}$ antigen emulsified in equal volume of incomplete Freund's adjuvant (IFA), (Sigma). The first booster dose was two weeks after the primary dose. The following booster doses were given at weekly intervals (Fagbemi, 1995; Guabadia and Fagbemi, 1997). Rabbit was sacrificed three days after the last dose and it's serum was obtained and pAb fraction was purified by $50 \%$ ammonium sulfate precipitation method (Nowo-tny, 1979). More purification of pAb was done by $7 \%$ caprilic acid method
(Mckinney and Parkinson, 1987). Anti-Giardia $\mathrm{IgG}$ pAb was kept at $-20^{\circ} \mathrm{C}$ till needed. 2- Conjugation of anti-Giardia IgG polyclonal antibodies with Horseradish Peroxidase (HRP): Five mg HRP, (Sigma) was resuspended in $1.2 \mathrm{ml}$ distilled water followed by addition of $0.3 \mathrm{ml}$ freshly prepared sodium periodate and incubation at room temperature for $20 \mathrm{~min}$. HRP solution was dialyzed against $1 \mathrm{mM}$ sodium acetate buffer $(\mathrm{pH} 4)$ at $4^{\circ} \mathrm{C}$ overnight (Tijssen and Kurstak, 1984). Anti-Giardia IgG pAb solution $(5 \mathrm{mg} / \mathrm{ml}$ in $0.02 \mathrm{M}$ carbonate buffer, $\mathrm{pH}$ 9.6) was prepared. The HRP was removed from dialysis tube and was added to $0.5 \mathrm{ml}$ of antibody solution. The mixture was incubated at room temperature for $2 \mathrm{~h}, 100 \mu \mathrm{l}$ sodium borohydride was added and the solution was incubated at $4^{\circ} \mathrm{C}$ for $2 \mathrm{~h}$. HRP conjugate $\mathrm{pAb}$ was dialyzed with several changes against 0.01 M PBS (pH 7.2).

3- Conjugation of anti-Giardia IgG polyclonal antibodies with graphene nanoparticles (GrNPs): Conjugation of antibodies to the GrNPs based on following protocol: Five ml GrNPs aqueous solution was diluted by 2 in PBS, and then bath sonicated for $1 \mathrm{~h}$ to make a clear solution. $1.2 \mathrm{~g} \mathrm{NaOH}$ and $1.0 \mathrm{~g}$ chloricacetic acid (Cl-CH2-COOH) was added into the $10 \mathrm{ml} \mathrm{GrNps}$ suspension $(2 \mathrm{mg} / \mathrm{ml})$ and bath sonicated for 1-3 h to convert the $-\mathrm{OH}$ groups to $-\mathrm{COOH}$ via conjugation of acetic acid moieties (named GrNps-COOH). The GrNps-COOH solution was neutralized and purified by rinsing and filtration. GrNps$\mathrm{COOH}$ suspension was diluted by water to optical density $\mathrm{OD}=0.4$ at $808 \mathrm{~nm}(1 \mathrm{~mm}$ optical path). $2 \mathrm{mg} / \mathrm{ml}$ pAb was added to $\mathrm{GrNps}$ $-\mathrm{COOH}$ suspension \& sonicated for $5 \mathrm{~min}$. N-(3-Dimethylam-inopropyl-N'-ethylcarbodiimide hydrochloride (EDC, Sigma Inc.) was added twice to reach $4 \mathrm{mM}$, reaction overnight, and quenched by Mercapto-ethanol (Fluka Inc.). Final product (GrNps-Ab) was obtained by ultra-centrifugation at $45 \mathrm{k}$ rpm in $2 \times$ phosphate buffered saline (PBS) for $1 \mathrm{~h}$ to save supernatant (yield $\sim 50 \%$ ).

Application of the prepared antigen and 
polyclonal antibodies in immunological techniques:

1- Preparation of Fecal Samples (Mezo et al, 2004): Individual fecal samples were processed by mixing the fecal material in a 1:9 proportion with PBS. Samples were mixed using a vortex to form slurry then centrifuged at $3000 \mathrm{rpm}$ for $30 \mathrm{~min}$ at $25^{\circ} \mathrm{C}$. Supernatant was recovered and stored at $-20^{\circ} \mathrm{C}$ until used. This step was done to make a solution (containing parasite antigens) from each faecal sample to be tested by the immunological methods.

2- Detection of Giardia Antigen in stool samples by Home-Made Sandwich ELISA: After several optimization trials of sandwich ELISA to detect the optimum dilution of coating $\mathrm{Ab}$ and detecting $\mathrm{Ab}$ (Venkatesan and Wakelin, 1993): Micro titer plates were coated with $100 \mu \mathrm{l} /$ well of purified IgG pAb as a capture antibody at dilution of $1 / 50$ in $0.1 \mathrm{M}$ carbonate buffer, $\mathrm{pH} 9.6$ and the plates were incubated at $4^{\circ} \mathrm{C}$ overnight. Plates were washed 3 times with washing buffer $0.1 \mathrm{M} \mathrm{PBS} / \mathrm{T}, \mathrm{pH}$ 7.4. Free sites were blocked with $200 \mu \mathrm{l} /$ well of blocking buffer $(0.1 \% \mathrm{BSA}$ in $0.1 \mathrm{M} \mathrm{PBS} / \mathrm{T})$ and incubated for $2 \mathrm{~h}$ at $37^{\circ} \mathrm{C}$. Plates were washed with washing buffer 3 times. $100 \mu$ l of fecal samples were added to each well and incubated for $2 \mathrm{~h}$ at $37^{\circ} \mathrm{C}$ and plates were washed 3 times with washing buffer. $100 \mu \mathrm{l} /$ well of anti-Giardia IgG pAb conjugated with HRP was added at dilution of $1 / 200$ in $0.1 \mathrm{M}$ PBS to all wells and plates were incubated for $1 \mathrm{~h}$ at $37^{\circ} \mathrm{C}$. Plates were washed 5 times with washing buffer, $100 \mu \mathrm{l}$ of substrate solution [a tablet of O-phenylenediamine dihydrochloride (OPD, Sigma)] dissolved in $25 \mathrm{ml}$ of $0.05 \mathrm{M}$ phosphate citrate buffer, $\mathrm{pH} 5$ with peroxidase $\mathrm{H}_{2} \mathrm{O}_{2}$, were added to each well and plates were incubated in dark place at room temperature for $30 \mathrm{~min}, 50 \mu \mathrm{l} /$ well of 8 $\mathrm{N} \mathrm{H} 2 \mathrm{SO} 4$ were added to stop the enzyme substrate solution. Absorbance was measured at $492 \mathrm{~nm}$ using ELISA reader (BioRad microplate, Richomond, Co.).

3- Detection of Giardia Antigen in stool samples by Home-Made Nano graphene based Sandwich ELISA: Micro titer plates were coated with $100 \mu \mathrm{l} /$ well of purified $\mathrm{IgG}$ $\mathrm{pAb}$ conjugated with Nano graphene as a capture antibody at dilution of $1 / 200$ in $0.1 \mathrm{M}$ carbonate buffer, $\mathrm{pH} 9.6$ and plates were incubated at $4{ }^{\circ} \mathrm{C}$ overnight. Steps were completed as mentioned in sandwich ELISA.

4- Detection of Giardia antigen in stool samples by Home-Made Dot-ELISA (Yamamoto et al, 1998): a. Five $\mu 1$ of the purified anti-Giardia IgG-pAb (coating antibody) diluted in carbonate buffer (1/50) was dotted on nitrocellulose (NC) membrane discs and allowed to air dry thoroughly. They were incubated at $4{ }^{\circ} \mathrm{C}$ overnight. b. In next morning, membrane was washed 3 times with PBS/T. Then blocking solution was applied, incubated at room temperature for $45 \mathrm{~min}$. After that the membrane was washed 3 times with PBS/T. Stool samples $(5 \mu 1)$ were dotted on NC discs then incubated for 30min and washed 3 times with PBS/T. c. Five $\mu l$ of HRP conjugated anti-Giardia IgG-pAb (detecting antibody) diluted in PBS buffer (1/200) was dotted on NC discs and incubated for 30min, then washed 5 times with PBS /T. d. DAB (Diamino-Benzidine tetrahydrocholoride) substrate $(0.5 \mathrm{mg} / \mathrm{ml}$ DAB $0.03 \%$ $\mathrm{H}_{2} \mathrm{O}_{2}$ in PBS) was applied by dotting $5 \mu \mathrm{l} /$ disc. NC discs that gave visible brown spots were considered positive. Reaction was stopped with cold distilled $\mathrm{H}_{2} \mathrm{O}$ just after color development.

5- Detection of Giardia antigen in stool samples by Home-Made Nano grapheme based Dot-ELISA: As with Dot ELISA but using Nano graphene conjugated PAb (at dilution of 1/200 in carbonate buffer) as coating antibodies and HRP conjugated PAb as detecting antibodies.

Statistical analysis: Data were presented as mean \pm standard deviation $(\mathrm{SD})$ of $(\mathrm{X} \pm \mathrm{SD})$. Cut off value $=$ mean $\mathrm{OD}$ readings of negateve controls+2SDs of mean. Sensitivity $(\%)=$ $\mathrm{A} /(\mathrm{A}+\mathrm{C}) \mathrm{x} 100$, specificity $(\%)=\mathrm{D} /(\mathrm{B}+\mathrm{D}) \mathrm{x}$ $100, \operatorname{PPV}(\%)=\mathrm{A} /(\mathrm{A}+\mathrm{B}) \times 100 \& \mathrm{NPV}(\%)=$ 
$\mathrm{D} /(\mathrm{C}+\mathrm{D}) \mathrm{x} 100$, as $\mathrm{A}=$ true positive, $\mathrm{B}=$ false positive, $\mathrm{C}=$ false negative $\& \mathrm{D}=$ true negative.

\section{Results}

Stool examination of 43 samples positive to Giardia cyst by different parasitological methods (direct smear, Mini Parasep \& MIFC) showed that all samples (43) were positive to Giardia cyst by Mini Parasep and MIFC methods (Figs. 4\&5). But, by using direct smear method, 41 samples were positive to Giardia cyst (Fig.3).

Comparison of the diagnostic performance of these methods (direct smear, Mini Parasep \& MIFC) used for detecting Giardia cyst in stool samples of GIa (Tab. 1, Chart 1) showed that Mini Parasep concentration technique was the best followed by MIFC technique. The least result was obtained from direct smear. This table shows statistically significant difference between them with the highest mean number of Giardia cyst in 3 different microscopic fields that was recorded from Mini Parasep technique (7.78) followed by MIFC technique (5.86) and direct smear method (3.52).

Assessment of Giardia antigen reactivity by rapid diagnostic test (Fig. 1) showed that Giardia antigen was reactive only against Giardia Ab (detected by presence of 2 bands; control and test bands) while it was not reactive against Cryptosporidium and $E$. histolytica/dispar Abs (detected by presence of one band; control band).

Total protein content of Giardia antigen was $5.1 \mathrm{mg} / \mathrm{ml}$ as measured by Bradford method. Protein content of anti-Giardia IgG Polyclonal antibodies: Total protein content was $1.8 \mathrm{mg} / \mathrm{ml}$ as measured by Bradford method after purification by $50 \%$ ammonium sulphate and dropped to $1.4 \mathrm{mg} / \mathrm{ml}$ after $7 \%$ caprylic acid purification method of rabbit's serum.

Traditional Sandwich ELISA for detection of Giardia antigen in stool samples (Tab. 2), showed 35 positive cases $(57.4 \%)$ in GI out of 61 . In GII, 8 cases were positive out of 20 while 12 cases were negative. In GIII, all

cases were negative, and calculated cut off value was 0.44 .

Nano graphene based Sandwich ELISA to detect Giardia antigen in stool samples (Tab, 3), showed 51 positive cases $(83.6 \%)$ in GI out of 61. In GII, 10 cases were positive out of 20 and 10 cases were negative. In GIII all cases were negative. Calculated cut off value was 0.43 .

Nano graphene based Sandwich ELISA was higher than Traditional Sandwich ELISA on detecting Giardia antigen in stools (Tab. 4) as to sensitivity ( $83.6 \%$ vs. $57.4 \%$ ), PPV (83.6\% vs. $81.4 \%)$, NPV (71.4\% vs. $50.9 \%)$ and diagnostic accuracy $(79.2 \%$ vs. 64.6\%). Traditional Sandwich ELISA was higher than Nano graphene based Sandwich ELISA; specificity ( $77.1 \%$ vs. $71.4 \%$ ), with significant difference (p- $<0.05$ ).

Dot ELISA for Giardia antigen in stool samples (Tab. 5) showed 56 positive cases $(91.8 \%)$ in GI out of 61. In GII, 7 cases were positive out of 20 and 13 cases were negative. In GIII all cases were negative.

Nano graphene based Dot ELISA for detection of Giardia antigen in stool samples (Tab. 6), showed 56 positive cases $(91.8 \%)$ in GI out of 61. In GII, 11 cases were positive out of 20 and 9 cases were negative. In GIII all cases were negative.

Dot ELISA and Nano graphene based Dot ELISA had same sensitivity $(91.8 \%)$ for $G i$ ardia antigen in stool samples (Tab. 7). Dot ELISA was higher than Nano graphene based Dot ELISA as to specificity ( $80 \%$ vs. $68.6 \%)$, PPV (88.9\% vs. $83.6 \%)$, NPV $(84.8 \%$ vs. $82.8 \%)$ and diagnostic accuracy ( $87.5 \%$ vs. $83.3 \%)$, but without significant difference ( $\mathrm{p}$-value $>0.05$ ).

\section{Results}

The results were in tables and figures 
Table 1: Comparison of the diagnostic performance of different methods (direct smear, Mini Parasep \& MIFC) used for detecting Giardia cyst in stool samples of GIa using mean \pm SD and ANOVA test

\begin{tabular}{|l|c|c|c|c|c|c|c|}
\hline & \multicolumn{4}{|c|}{ Giardia cyst under microscope } & \multicolumn{2}{c|}{ ANOVA } \\
\cline { 2 - 7 } & Positive & Mean & \pm SD & Min. & Max. & F & p-value \\
\hline Direct & 41 & 3.52 & 3.14 & 0.67 & 12 & & \multirow{2}{*}{6.167} \\
MIFC & 43 & $5.86(\mathrm{a})$ & 6.23 & 0.3 & 30 & $0.03 *$ \\
\hline Mini Parasep. & 43 & $7.78(\mathrm{a}, \mathrm{b})$ & 6.58 & 1 & 25 & & \\
\hline
\end{tabular}

a: significant difference direct $<0.05$, b: significant difference MIF $<0.05$

Table 2: Detection of Giardia antigen in stool samples using Traditional Sandwich ELISA in groups

\begin{tabular}{|l|c|c|c|c|c|c|}
\hline \multirow{2}{*}{ Groups } & \multicolumn{2}{|c|}{ Cut-off=0.44: Traditional Sandwich ELISA } & \multicolumn{2}{c|}{ Total } \\
\cline { 2 - 6 } & \multicolumn{2}{|c|}{ Positive } & \multicolumn{2}{c|}{ Negative } & \multicolumn{2}{c|}{$\%$} \\
\cline { 2 - 6 } & No. & $\%$ & No. & $\%$ & No. & $\%$ \\
\hline Group I: Giradia & 35 & $57.40 \%$ & 26 & $42.60 \%$ & 61 & $100.00 \%$ \\
\hline a: Cyst & 27 & $55.1 \%$ & 22 & $44.9 \%$ & 49 & $100.0 \%$ \\
\hline b: Trophozoite & 8 & $66.7 \%$ & 4 & $33.3 \%$ & 12 & $100.0 \%$ \\
\hline Group II: Other parasites & 8 & $40.0 \%$ & 12 & $60.0 \%$ & 20 & $100.0 \%$ \\
\hline Group III: Healthy Control & 0 & $0.0 \%$ & 15 & $100.0 \%$ & 15 & $100.0 \%$ \\
\hline
\end{tabular}

Table 3: Detection of Giardia antigen in stool samples by Nano graphene based sandwich ELISA in groups

\begin{tabular}{|l|c|c|c|c|c|c|}
\hline \multirow{2}{*}{ Groups } & \multicolumn{2}{|c}{ Cut-off=0.43: Nano Sandwich ELISA } & \multicolumn{2}{c|}{ Total } \\
\cline { 2 - 7 } & \multicolumn{2}{|c|}{ Positive } & \multicolumn{2}{c|}{ Negative } & \multicolumn{2}{c|}{$\%$} \\
\cline { 2 - 7 } & No. & $\%$ & No. & $\%$ & No. & $\%$ \\
\hline Group I: Giradia & 51 & $83.60 \%$ & 10 & $16.40 \%$ & 61 & $100.00 \%$ \\
\hline a: Cyst & 40 & $81.6 \%$ & 9 & $18.4 \%$ & 49 & $100.0 \%$ \\
\hline b: Trophozoite & 11 & $91.7 \%$ & 1 & $8.3 \%$ & 12 & $100.0 \%$ \\
\hline Group II: Other parasites & 10 & $50.0 \%$ & 10 & $50.0 \%$ & 20 & $100.0 \%$ \\
\hline Group III: Healthy Control & 0 & $0.0 \%$ & 15 & $100.0 \%$ & 15 & $100.0 \%$ \\
\hline
\end{tabular}

Table 4: Sensitivity, specificity, PPV, NPP and diagnostic accuracy percentage of Traditional Sandwich ELISA and Nano graphene based Sandwich ELISA for detection of Giardia antigen in stool samples

\begin{tabular}{|l|l|l|l|l|l|}
\hline Techniques & Sens. & Spec. & PPV & NPV & Accuracy \\
\hline Traditional Sandwich ELISA & $57.4 \%$ & $77.1 \%$ & $81.4 \%$ & $50.9 \%$ & $64.6 \%$ \\
\hline Nano Sandwich ELISA & $83.6 \%$ & $71.4 \%$ & $83.6 \%$ & $71.4 \%$ & $79.2 \%$ \\
\hline
\end{tabular}

Table 5: Detection of Giardia antigen in stool samples using Dot ELISA in groups

\begin{tabular}{|l|c|c|c|c|c|c|}
\hline \multirow{2}{*}{ Dot ELISA } & \multicolumn{2}{|c|}{ Positive Cases } & \multicolumn{2}{|c|}{ Negative Cases } & \multicolumn{2}{c|}{ Total } \\
\cline { 2 - 7 } & No. & $\%$ & No. & $\%$ & No. & $\%$ \\
\hline Group I: Giradia & 56 & $91.8 \%$ & 5 & $8.2 \%$ & 61 & $100 \%$ \\
\hline a: Cyst & 47 & $95.9 \%$ & 2 & $4.1 \%$ & 49 & $100 \%$ \\
\hline b: Trophozoite & 9 & $75.0 \%$ & 3 & $25.0 \%$ & 12 & $100 \%$ \\
\hline Group II: Other parasites & 7 & $35.0 \%$ & 13 & $65.0 \%$ & 20 & $100 \%$ \\
\hline Group III: Healthy Control & 0 & $0.0 \%$ & 15 & $100.0 \%$ & 15 & $100 \%$ \\
\hline
\end{tabular}

Table 6: Detection of Giardia antigen in stool samples using Nano geaphene based Dot ELISA in groups

\begin{tabular}{|l|c|c|c|c|c|c|}
\hline \multirow{2}{*}{ Nano Dot ELISA } & \multicolumn{2}{|c|}{ Positive Cases } & \multicolumn{2}{|c|}{ Negative Cases } & \multicolumn{2}{|c|}{ Total } \\
\cline { 2 - 7 } & No. & $\%$ & No. & $\%$ & No. & $\%$ \\
\hline Group I: Giradia & 56 & $91.8 \%$ & 5 & $8.2 \%$ & 61 & $100 \%$ \\
\hline a: Cyst & 45 & $91.8 \%$ & 4 & $8.2 \%$ & 49 & $100 \%$ \\
\hline b: Trophozoite & 11 & $91.7 \%$ & 1 & $8.3 \%$ & 12 & $100 \%$ \\
\hline GII: Other parasites & 11 & $55.0 \%$ & 9 & $45.0 \%$ & 20 & $100 \%$ \\
\hline GIII: Healthy Control & 0 & $0.0 \%$ & 15 & $100.0 \%$ & 15 & $100 \%$ \\
\hline
\end{tabular}

Table 7: Sensitivity, specificity, PPV, NPP and diagnostic accuracy percentage of Dot ELISA and Nano geaphene based Dot ELISA for detection of Giardia antigen in stool samples

\begin{tabular}{|l|c|c|c|c|c|}
\hline Technique & Sensitivity & Specificity & PPV & NPV & Accuracy \\
\hline Dot ELISA & $91.8 \%$ & $80.0 \%$ & $88.9 \%$ & $84.8 \%$ & $87.5 \%$ \\
\hline Nano Dot ELISA & $91.8 \%$ & $68.6 \%$ & $83.6 \%$ & $82.8 \%$ & $83.3 \%$ \\
\hline
\end{tabular}




\section{Discussion}

Microscopic techniques (direct or concentrated) for fecal samples are still commonly used to detect Giardia cysts or trophozoites. But, analysis of single stool sample and the skill of the microscopist can affect the detected accuracy (Beyhan and Cengiz, 2017). Given these difficulties the development of sensitive, cost effective and rapid diagnostic methods is of the most importance (Moharam et al, 2014).

Single antigen detection technique detected $50 \%$ more infections than the routine stool examination (Garcia, 2007).

Nanotechnology may improve sensitivity, selectivity, speed, cost, and convenience of diagnostic tests (Hegazy et al, 2015).

The present study revealed that Mini Parasep concentration technique was the best followed by MIFC technique and the least result was obtained from direct smear. There is a statistically significant difference between them.

Sanprasert et al. (2016) showed that Mini Parasep ${ }^{\circledR}$ SF is the most sensitive $(56.38 \%)$ in the intestinal parasites detection among school-age children, followed by direct smear $(40.43 \%)$ and modified formol ether concentration technique (M-FECT) (37.32\%).

Zeeshan et al. (2011) found that Parasep filters enhanced the ability to detect intestinal parasites in stool; 100 samples were negative for parasites using direct microscopy whereas 13 of them were positive with Parasep.

On the other hand, Kitvatanachai and Rhongbutsri (2017) recorded the highest efficacy of direct smear technique in detecting intestinal parasites (74.62\%), followed by modified formol ether concentration technique $(65.67 \%)$ and Mini Parasep ${ }^{\circledR}$ SF kit (55.22\%).

The present study revealed that microscopic examination was simple, non-invasive and allowed the detection of other parasitic infections. This agreed with Lebwohl et al. (2003). However, routine microscopic examination for diagnosis of giardiasis is time consuming and relies on the microscopist's skills and experience (Rosoff et al, 1989; Scheffler and Van Etta, 1994; Schuurman et $a l, 2007)$. The sensitivity of laboratory diagnosis of Giardia infection can be improved by repeating stool examination on 3 consequative days. But this was not possible in the present study, as the patients were attending the outpatient clinics.

In order to increase the sensitivity, different immunological methods have been developed as an alternative for the diagnosis of giardiasis (Chakarova, 2010).

In this study, a novel antigen-capture immunoassay based on IgG pAb conjugated with graphene nanoparticles was used for detection of Giardia antigen in stool samples which was used as a first trial for diagnosis of human giardiasis.

The current study (Tab. 4) demonstrated that Nano Sandwich ELISA was superior to Traditional Sandwich ELISA on detection of Giardia antigen in stool samples regarding sensitivity ( $83.6 \%$ vs. $57.4 \%)$, PPV $(83.6 \%$ vs. $81.4 \%)$, NPV (71.4\% vs. $50.9 \%)$ and diagnostic accuracy (79.2\% vs. $64.6 \%)$, while specificity in Traditional Sandwich ELISA was higher than Nano Sandwich ELISA (77.1\% vs. $71.4 \%)$. This was a statistically significant difference between them $(\mathrm{P}<$ 0.05). This means that the use of graphene nanoparticles improved the diagnostic testing of human giardiasis.

Moharam et al. (2014) compared Nano gold based ELISA and Traditional Sandwich ELISA in detecting Giardia antigen in stool samples; they reported that the sensitivity and specificity of Nano Sandwich ELISA was higher than that of Traditional Sandwich ELISA $(95.8 \%$ vs. $93 \%$ \& $95 \%$ vs. $92.5 \%)$ respectively.

Another study aimed was to detect potential specificity and sensitivity of paramagnetic nanoparticles based ELISA for diagnosis of human giardiasis by detection of $\mathrm{Gi}^{-}$ ardia copro-antigen, Koura et al. (2016) reported that Sandwich ELISA achieved sensitivity of $88 \%$ and specificity of $92 \%$, while 
immuno-magnetic bead ELISA with paramagnetic nanoparticles achieved higher values of sensitivity and specificity; 92\% \& $94 \%$, respectively.

The difference between the results of the present work and other studies may be explained by the difference in the method of antigen preparation, the strain of Giardia and the number of participating individuals in each study; positive cases, other parasites and control groups.

Several studies compared between Nano based ELISA versus (vs.) Traditional ELISA for detection of parasitic infection. Among them, Naser et al. (2017) who compared the sensitivity and specificity of the Traditional Sandwich ELISA vs. Nano gold based Sandwich ELISA for detection of Cryptosporidium antigen in stool samples. They reported that the sensitivity of Nano gold based Sandwich ELISA was higher than that of Traditional Sandwich ELISA (85\% vs. $68 \%$ ), while the specificity of Nano gold based Sandwich ELISA was lower than that of Traditional Sandwich ELISA (73.4\% vs. 77.3\%). Rashed et al. (2018) tested the Validity of Sandwich ELISA and Nano-gold Sandwich-ELISA for diagnosis of human hydatidosis. They reported that Nano-gold Sandwich-ELISA had higher values than Traditional Sandwich-ELISA regarding sensitivity (96.3\% vs. $81.5 \%$ ); specificity (95\% vs. $80 \%)$; PPV (96.3\% vs. $84.6 \%)$; NPV (95\% vs. $88.89 \%)$ and diagnostic accuracy (95.7\% vs. $80.9 \%$ ).

The current work showed that Dot ELISA and Nano graphene based Dot ELISA had the same sensitivity (91.8\%) on detecting

\section{References}

Adam, RDA, 2001: Biology of Giardia intestinalis. Clin. Microbiol. Rev. 14:447-5.

Aly, I, Zalat, R, El Aswad, BW, Moharm, IM, Masoud, BM, et al, 2013: Novel nanomagnetic beads based-latex agglutination assay for rapid diagnosis of human schistosomiasis haematobium. Int. J. Med. H. Pharma. Bio. Med. Eng. 7, 12:708714.
Giardia antigen in stool samples while Dot ELISA was higher than Nano Dot ELISA on detecting Giardia antigen in stool samples regarding specificity ( $80 \%$ vs. $68.6 \%)$, PPV (88.9\% vs. $83.6 \%)$, NPV ( $84.8 \%$ vs. $82.8 \%)$ and diagnostic accuracy ( $87.5 \%$ vs. $83.3 \%$ ), but there is no statistically significant difference between them $(\mathrm{P}>0.05)$.

Referring to hydatidosis diagnosis; Rashed et al. (2018) tested the validity of DotELISA and Nano-gold Dot-ELISA for detection of human hydatidosis. They reported that Nano-gold Dot-ELISA had higher values than Traditional Dot-ELISA regarding sensitivity $(95.7 \%$ vs. $88.9 \%)$; specificity (95\% vs. $80.7 \%$ ); PPV (96.3\% vs. $85.7 \%$ ); NPV (95\% vs. $84.2 \%$ ) and diagnostic accuracy (96.3 vs. $85.1 \%$ ).

The low sensitivity and specificity by using ELISA methods in the current work than some other studies may be due to dilution of stool samples during the preparation, which may be not adequate for carrying enough number of the cysts/trophozoites to be detected especially in cases with light infection; when few numbers are present in the specimen. This agreed with Naser et al. (2017).

\section{Conclusion}

Microscopic examination is reliable in diagnosis of human giardiasis as a first choice especially concentration techniques such as Mini Parasep and MIFC. In addition, this study using the prepared anti-Giardia $\mathrm{IgG}$ $\mathrm{pAb}$ that was carried out for detection of $G i$ ardia antigen in stool samples of patients infected with Giardia revealed that the use of graphene nanoparticles improved the diagnostic testing of human giardiasis.

Arora, DR, Arora, BB, 2015: Medical parasitology $3^{\text {rd }}$ ed. 41-45.CBS publisherd distributors PVT.LTD. New Delhi. Bangalore. Pune. Cochin. Chennai (India) 41:46-9.

Barazesh, A, Majidi, J, Fallah, E, 2010: Designing of enzyme linked immunosorbent assay (ELISA) kit for diagnosis copro-antigens of Giardia lamblia. Afri. J. Biotechnol. 9, 31:5025-7. 
Beyhan, YE, Cengiz, ZT, 2017: Comparison of microscopy, ELISA, and real-time PCR for detection of Giardia intestinalis in human stool specimens. Turk. J. Med. Sci. 47:1295-9.

Blagg, W, Mansour, NS, Khalaf, GL, 1955: A new concentration technique for the demonstration of protozoa and helminthic eggs in feces. Am. J. Trop. Med. Hyg. 4:23-8.

Bradford, MM, 1976: A rapid and sensitive method for the quantitation of microgram quantities of protein utilizing the principle of protein-dye binding. Anal. Biochem. 72, 4:245-54.

Caccio, SM, Sprong, H, 2011: Epidemiology of giardiasis in humans. In: Lujan HD, Svard S, editors. Giardia: A Model Organism. Vienna, Austria: Springer-Verlag.

Chakarova, B, 2010: comparative evaluation of the diagnostic methods for detection of Giardia intestinalis in human fecal samples. Trakia J. Sci. 8, 2: 174-9.

Diagbouga, S, Kientega, T, Erismann, S, Ouermi, D, Saric, J, et al, 2017: Evaluation of a Real-Time Polymerase Chain Reaction for the Laboratory Diagnosis of Giardia intestinalis in Stool Samples from Schoolchildren from the Centre-Ouest and Plateau Central Regions of Burkina Faso. Appl. Micro. Open Access. 3, 1:126-33.

Duque-Beltrán, S, Nicholls-Orejuela, RS, Jamaica, A, Guerrero-Lozano, R, Montenegro, S, et al, 2002: Detection of Giardia duodenalis antigen in human fecal eluates by enzyme-linked immunosorbent assay using polyclonal antibodies. Mem. Inst. Oswaldo. Cruz; Rio de Janeiro. 97: 1165-8.

Eissa, MM, Amer, EI, 2010: Giardia lamblia: A new target for miltefosine .Int. J. Parasitol. 42, 5: 443-52.

El-Deeb, MT, Abdel-Hamid, DH, 2012: Growth assessment in Egyptian children with chronic diarrhea. J. Egypt. Soc. Parasitol. 42, 3:605-17.

El-Nahas, HA, Salem, DA, El-Henawy, AA, ElNimr, HI, Abdel Ghaffar HA, et al, 2013: Giardia diagnostic methods in human fecal samples: A comparative study. Cytometry B. Clin. Cytom. 84: 44-9.

Escobedo, AA, Hanevik, K, Almirall, P, Cimerman, S, Alfonso, M, 2014: Management of chronic Giardia infection. Expert. Rev. Anti. Infect. Ther. 12, 9:1143-57.

Fagbemi, BO, 1995: Development and characterization of a monoclonal antibody reactive with 128 $\mathrm{kDa}$ protease of $F$. gigantica. Vet. Parasitol. 57, 2: 351-4.

Garcia, LS, 2007: Intestinal protozoa, flagellates \& ciliates. In: Diagnostic Medical Parasitology. ASM Press, Washington DC; Part II.

Geurden, T, Vercruysse, J, Claerebout, E, 2010: Is Giardia a significant pathogen in production animals? Exp. Parasitol. 124:98-106.

Guobadia, EE, Fagbemi, BO, 1997: The isolation of $F$. gigantica specific antigens and their use in the serodiagnosis of fascioliasis in sheep by detection of circulating antigens. Vet. Parasitol. 68, 3:269-82.

Hanson, KL, Cartwright, CP, 2001: Use of an enzyme immunoassay does not eliminate the need to analyze multiple stool specimens for sensitive detection of Giardia intestinalis. J. Clin. Microbiol. 39: 474-7.

Hegazy, S, Farid, A, Rabae, I, 2015: Novel IMB-ELISA assay for rapid diagnosis of human toxoplasmosis using SAG1 antigen. Jpn. J. Infect. Dis. 68:474-80.

Heyworth, MF, 2014: Immunological aspects of Giardia infections. Parasite 21:55-9.

Hu, YDH, Fine, A, Tasciotti, EA, 2011: Nanodevices in dignostics. Wiley Interdiscip. Rev. Nanomed. Nanobiotechnol. 3, 1:11-32.

Kitvatanachai, S, Rhongbutsri, P, 2017: Using Mini Parasep SF to determine intestinal parasitic infections comparing to conventional methods in gardener of Chanthaburi Province, Thailand, Asian Pac. J. Trop. Dis. 7, 10:596-600.

Klotz, C, Aebischer, T, 2015: The immunological enigma of human giardiasis. Curr. Trop. Med. Rep. 2:119-27.

Koura, EA, Aly, IR, El-deeb, NI, Shahin, M A, 2016: Effective diagnosis of giardiasis (Giardia lamblia) by immunomagnetic bead ELISA technique using paramagnetic nanoparticles. World J. Pharm. Sci. 4, 6:346-56.

Lebwohl, B, Deckeibaum, RJ, Green, PH, 2003: Giardiasis. Gastrointest. Endosc. 57, 7: 906-13.

Mckinney, MM, Parkinson, A, 1987: A simple, non-chromatographic procedure to purify immunoglobulins from ascites fluid. J. Immunol. Meth. 96, 2:271-8.

Mendonça, FF, Sudré, AP, Macieira, DB, Almosny, NR, 2017: The influence of serial fecal sampling on the diagnosis of giardiasis in humans, dogs, and cats. Rev. Inst. Med. Trop. São. Paulo 59:1-6.

Mezo, M, Gonzalez, W, Carro, C, Ubeira, F, 2004: An ultra-sensitive capture ELISA for detection of Fasciola hepatica coproantigens in sheep and cattle using a new monoclonal antibody (MM3) J. Parasitol. 90:845-52.

Moharm, IM, El Enain, G, El Aswad, BW, Hen 
dawy, M, Aly, I, et al, 2014: Evaluation of Nanogold beads based-ELISA for detection of Giardia lamblia antigen in stool samples of infected patients. Middle-East J. Sci. Res. 21, 12: 2264-73.

Moss, DM, Mathews, HM, Visvesvara, GS, Dickerson, JW, Walker, EM, 1991: Purification and characterization of Giardia lamblia antigens in the feces of Mongolian gerbils. J. Clin. Microbiol. 29, 1:21-6.

Naser, ME, Younis, MS, Bayoumi, IR, Eraky, MA, Aly, NS, et al, 2017: Evaluation of Nano gold beads based ELISA for detection of Cryptosporidium antigen in stool samples of diarrheic patients. Benha Med. J. 34:88-92.

Nowotny, A, 1979: Basic Exercises in Immunochemistry. Springer Verlag, Berlin Heidelberg. N. Y.

Rashed, SM, Naser, ME, Bayoumi, IR, El-Awamy, W, Gomaa, AS, 2018: Evaluation of nanogold particles-based enzyme-linked immunnosorbent assay for detection of hydatidosis. Benha Med. J. 35: 134-8.

Romero, LG, Quintero, J, Garcia, AH, Velazquiz, C, 2015: Host defences against Giardia lamblia. Parasite Immunol. 37:394-406.

Rosoff, JD, Sanders, CA, Sonnad, SS, De Lay, P R, Hadley, WK, et al, 1989: Stool diagnosis of giardiasis using a commercially available enzyme immunoassay to detect Giardia specific antigen 65. J. Clin. Microbiol. 27, 9:1997-2002.

Sanprasert, V, Charuchaibovorn, S, Bunkasem, U, Srirungruang, S, Nuchprayoon, S, 2016: Comparison between direct smear, formalin-ethyl acetate concentration, and Mini Parasep solvent-free concentrator for screening of intestinal parasitic infections among school-age children. Chula Med. J. 60, 3: 255-69.

Scheffler, EH, van Etta, LL, 1994: Evaluation of rapid commercial enzyme immunoassay for detection of Giardia lamblia in formaline preserved stool specimens. J. Clin. Microbiol. 32, 7:1807-8.

Schuurman, T, Lankamp, P, van Belkum, A, Kooistra-Smid, M, van zwet, A, 2007: Comparison of microscopy, real-time PCR and a rapid immunoassay for the detection of Giardia lamblia in human stool specimens. Clin. Microbiol. Infect. 13, 12:1186-91.

Tijssen, P, Kurstak, P, 1984: Highly efficient and simple methods for the preparation of peroxidase \& active peroxidase-antibody conjugate for enzyme immunoassays. Anal. Biochem. 136, 2: 451-7.

Venkatesan, P, Wakelin, D, 1993: ELISA for parasitologists; or lies, damned lies and ELISA. Parasitol. Today 9, 2:228-32.

Yamamoto, YI, Mineo, JR, Meneghisse, CS, Guimarães AC, Kawarabayashi, M, 1998: Detection in human sera of IgG, IgM and IgA to excreted/ secreted antigens from Toxoplasma gondii by use of dot-ELISA and immunoblot assay. Ann. Trop. Med. Parasitol. 92, 1:23-30.

Zeeshan, M, Zafar, A, Saeed, Z, Irfan, S, Sobani, ZA, et al, 2011: Use of Parasep filter fecal concentrator tubes for the detection of intestinal parasites in stool samples under routine conditions. Indian J. Pathol. Microbiol. 54, 1:121-3.

\section{Explanation of figures}

Chart 1: comparison between direct smear, Mini Parasep \& MIFC techniques used for detecting Giardia cyst in stool samples of GIa Fig. 1: Mini parasep

Fig. 2: Assessment of reactivity of Giardia antigen by Rapid diagnostic test

Fig. 3: Giardia cyst, direct smear, stained with iodine (X1000)

Fig. 4: Giardia cyst (prepared with MIFC, X1000)

Fig. 5: Giardia cysts (prepared with Mini Parasep, unstained, X1000)

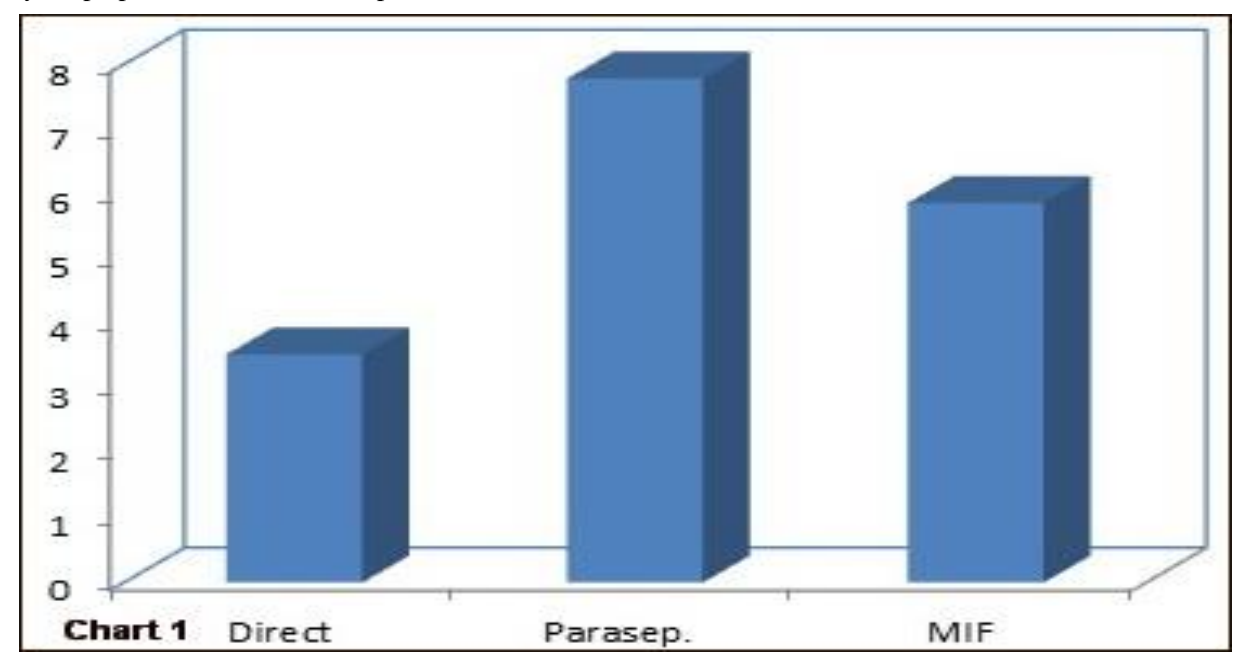



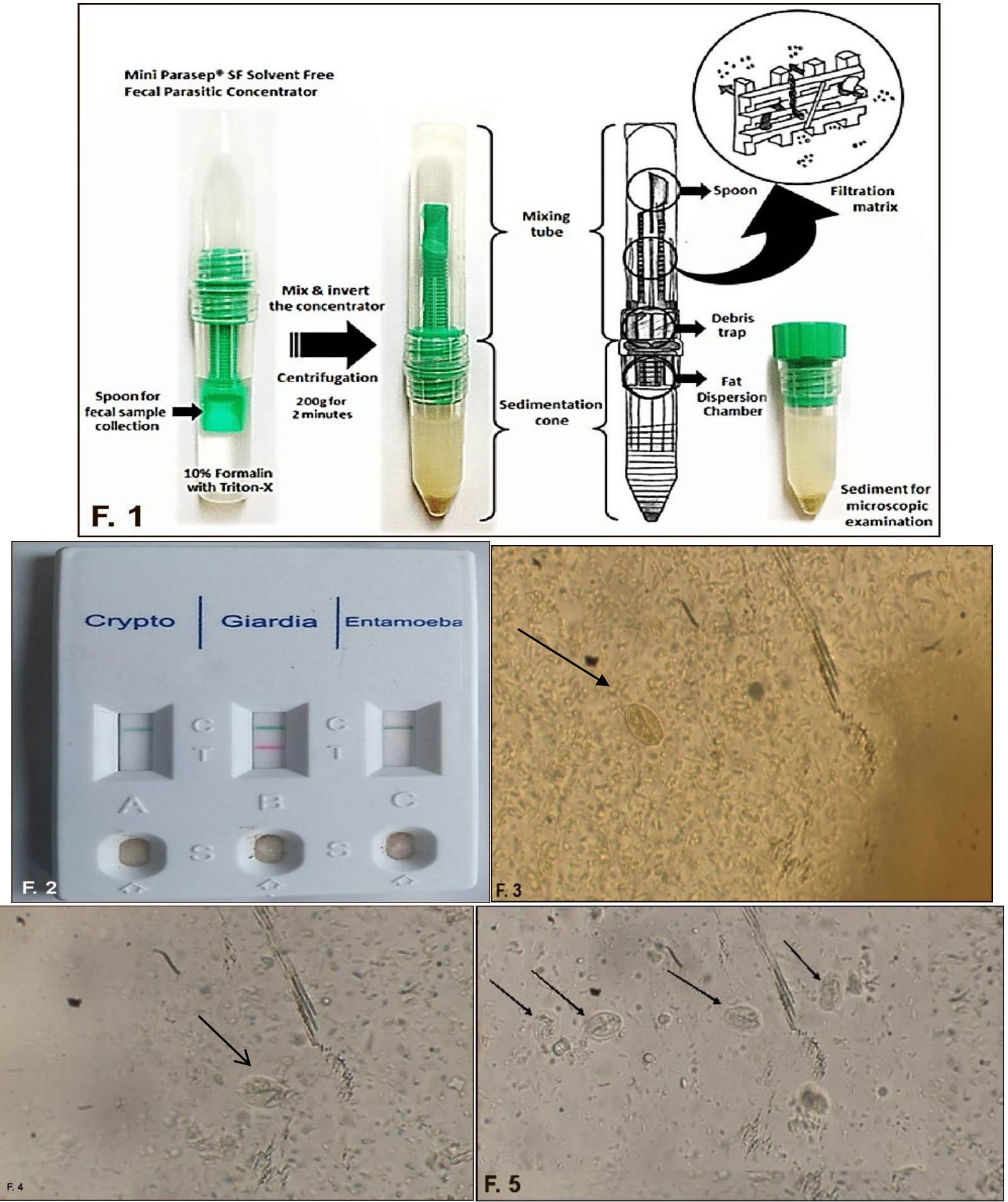